\title{
ISUP Grade Pattern 2
}

National Cancer Institute

\section{Source}

National Cancer Institute. ISUP Grade Pattern 2. NCI Thesaurus. Code C162655.

Like pattern 1, fairly circumscribed, yet at the edge of the tumor nodule there may be minimal infiltration; Glands are more loosely arranged and not quite as uniform as Gleason pattern 1. 